\title{
PHOTO-CATHODE PREPARATION SYSTEM OF THE A0 PHOTO-INJECTOR
}

\author{
M.Kuchnir, W. Hartung, E. Hahn \\ Fermilab \\ P. Michelato, D. Sertore \\ INFN, L.A.S.A. Milano
}

\section{INTRODUCTION}

This Technical Memorandum was written in Nov.1998 and was intended as part of a much larger report that has not been completed. It is being presented now because the material in it is of high utility for the infrequent but important operations involving preparation and rejuvenation of photo-cathodes.

The "A0 Photo-Injector" is an electron accelerator located in the AZero high bay area of Fermilab. A pulsed laser system generates electron bunches by the photo-electric effect when hitting a photo-cathode in a 1.5-cell, $1.3 \mathrm{GHz}$ RF gun. A 9-cell, 1.3 GHz superconducting resonant cavity then accelerates the electrons to $15 \mathrm{MeV}$. The $10 \mathrm{ps}$ time resolved waveform of the laser pulses is transferred to the electron bunches. This report is focused on the first hardware component of this accelerator, the Photo-cathode Preparation System. The reason for its existence is in the nature of the photo-electric material film used: $\mathrm{Cs}_{2} \mathrm{Te}$ (Cesium Telluride), a very reactive compound that once coated on the cathode requires that it be transported and used in ultra high vacuum (UHV), i.e.< $10^{-9}$ Torr.

The work leading to the selection of $\mathrm{Cs}_{2} \mathrm{Te}$ was carried out at CERN ${ }^{1}$, and Los Alamos $^{2}$ with a very relevant study carried out in European laboratories. A review of it has been presented by P. Michelato. The design of this system of UHV components was carried out in Milan ${ }^{5}$, along with the fabrication of its non-commercially-available parts. The Photo-cathode Preparation System at Fermilab is the first of two systems; the second (improved with the experience gained from the first) is to be used in the preparation and transportation of photocathodes for the TESLA Test Facility at DESY in Hamburg. Prior to the arrival of parts from Milan, the support structure was designed at Fermilab and built in its village machine shop. The assembly, leak checking and baking was carried out at Fermilab Lab 7 by the originators (Paolo Michelato, Daniele Sertore, Carlo Gesmundo) and some of the future local operators (Walter Hartung, Eileen Hahn and Moyses Kuchnir). In the process besides equipment transfer, we had transfer of the technology for photoemissive film deposition and some UHV techniques. The Photo-cathode Preparation System was then transported to A0 on Feb. 12, 1997 where a first cathode was made which yielded a typical $-11 \%$ quantum efficiency on Jun. 2, 1997. 


\section{APPARATUS DESCRIPTION}

Figure 1 shows the system in position in the $\mathrm{A} 0$ accelerator vault and Figure 2 shows the vacuum system in more detail. The chamber proper is a $M^{2} C^{6}$ catalog item as are the manipulators $\mathrm{A}^{7}, \mathrm{~B}^{8}, \mathrm{C}, \mathrm{D}$ and $\mathrm{E}^{9}$.

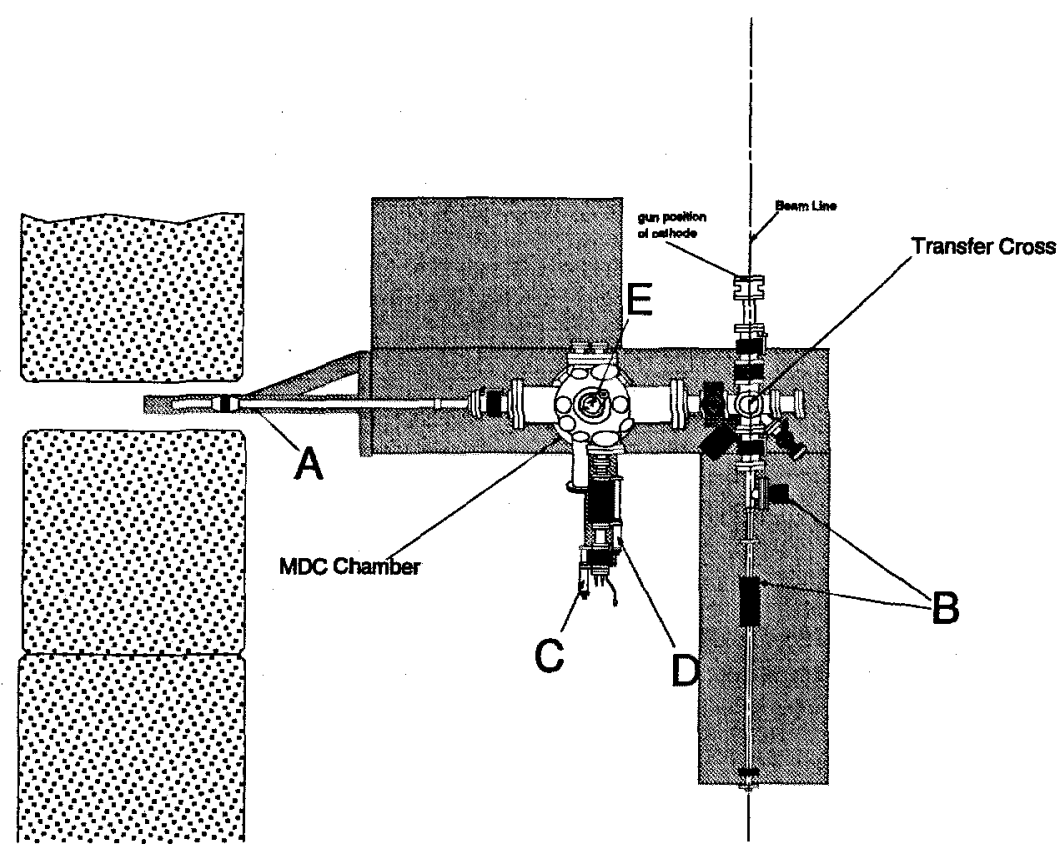

Figure 1. Cathode Preparation System and supporting structure in the A0 accelerator vault.

\section{BASIC CONSIDERATIONS}

A few obvious comments on ultra high vacuum (UHV) might be in order: Every material exposed to UHV has to be of low vapor pressure and it must be thoroughly cleaned and degreased (no fingerprints). The standard materials are Stainless Steel , and Copper (for gaskets). Special care is required to prevent contamination by back streaming of vacuum pump oil vapor: very clean and uncontaminated flexible metal hoses are used to connect the UHV system through a liquid Nitrogen trap to the turbo pump of the pumping system. The Viton " $O$ " ring used in these connections are not greased, since even vacuum grease is to be avoided. The vacuum is ultimately maintained by ion pumps and the pressure is measured with ionization gauges, a residual gas analyzer (RGA) or the ion pump controllers themselves. Exposure to air will cover the surfaces with layers of water, so that a vacuum baking operation is always needed for desorbing water and other gases that might be absorbed in the body of the materials or adsorbed on their surfaces. Once the ion pumps can operate without the turbo pump, the later is valved off and removed from the system. Every time the system is exposed to atmosphere, it must be baked again. This is costly in terms of time and manpower. It requires external heating tapes and regulators, as well as insulation with many layers of Aluminum foil, in addition to the actual baking time. So this is an operation to be avoided and, when needed, it should be done in planned stages that prevent exposing coated cathodes. One interesting observation about moving parts in UHV: friction generates gas (promptly indicated by the ion gauges) as well as dust particles. 
view from above table level:

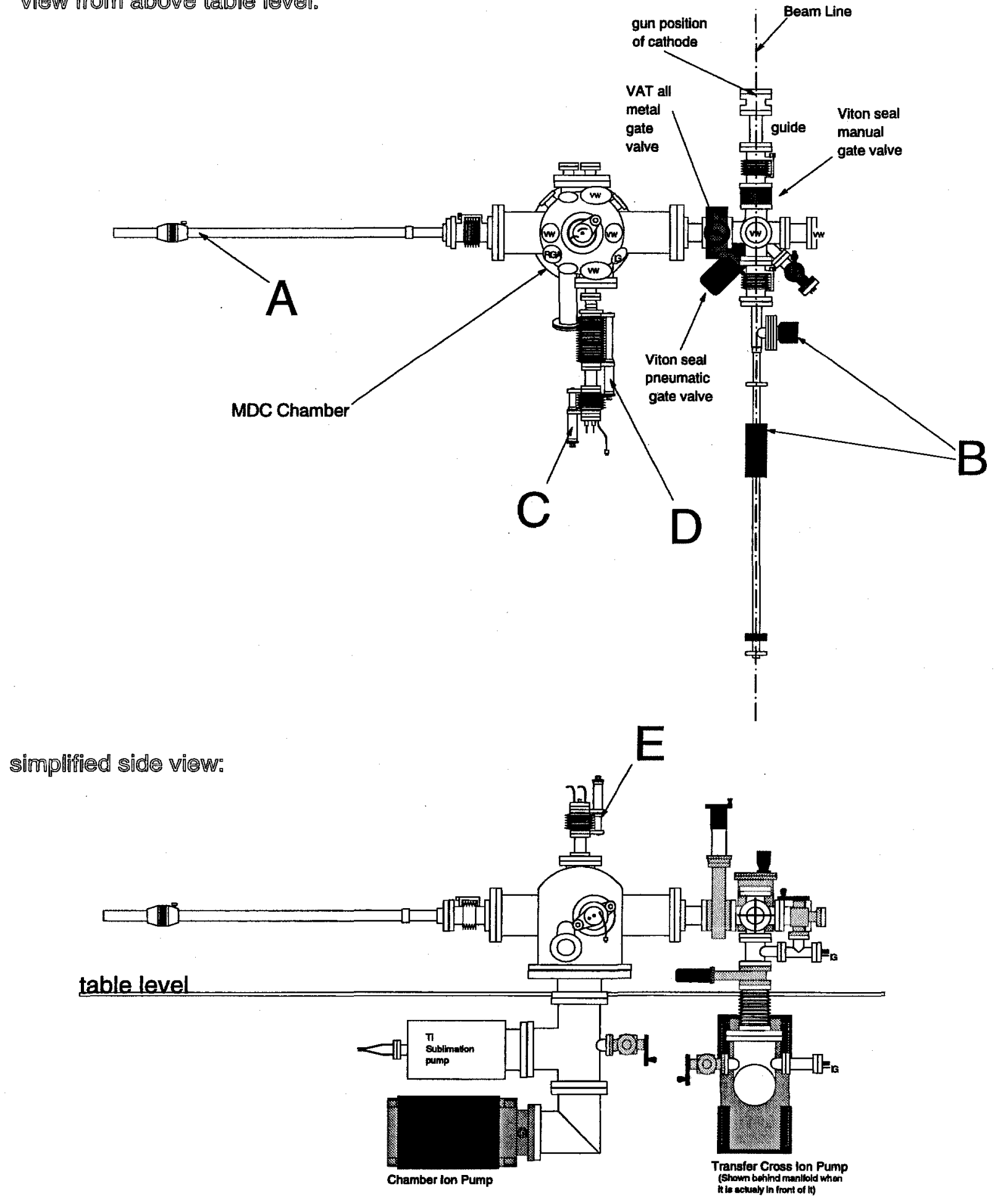

Figure 2. More detailed view of the Cathode Preparation System 
Manipulators A and B exert their mechanical action inside the vacuum by means of permanent magnets while $\mathrm{C}, \mathrm{D}$ and $\mathrm{E}$ operate via bellows. Manipulator A moves a storing carriage which can hold up to 5 cathodes.

The cathodes are machined from Molybdenum with a flat polished end where the $\mathrm{Cs}_{2} \mathrm{Te}$ is deposited. As shown in Figure 3, the cathode body is mostly cylindrical and hollow. Flats and grooves on the external surface of the cathode allow for rigid pincers to slide over and grab the photo-cathode body, while spring-loaded balls hold it in a well defined position, as shown in Figure 4. The carriage also has spring-loaded balls to secure the photo-cathodes.

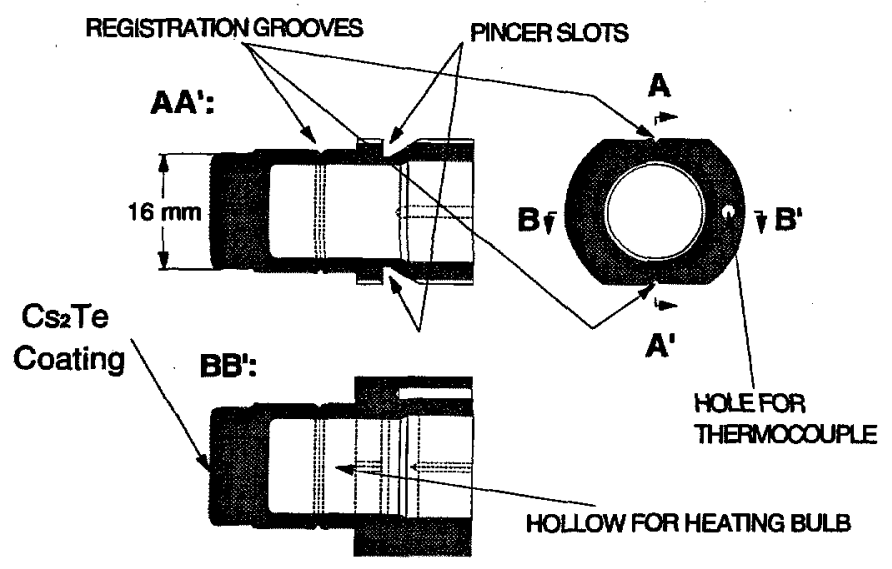

Figure 3. Three-view drawing of a photo-cathode body.

In the hollow of the cathode, one can insert a halogen bulb that serves as a heater. The bulb and a thermocouple (which fits in the hole alongside the hollow) are attached to the tip of manipulator $\mathrm{C}$, which rides inside manipulator $\mathrm{D}$.

Manipulator $\mathrm{D}$ has a hollow rigid pincer that can grab a cathode from the carriage and move it into the coating position and vise versa.

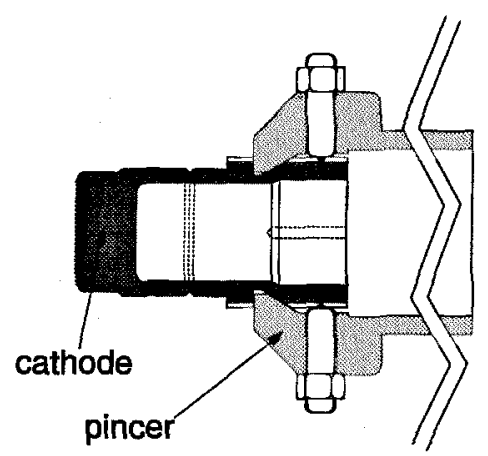

Figure 4. Pincer holding a cathode

Manipulator B has a rigid pincer that can grab a cathode from the carriage and move it along the beam line into its operating position in the gun and vice-versa. This manipulator (unlike A) can exert the considerable force necessary to insert the cathode into 
the toroidal spring that centers it in the gun. It has two handles, one for translation and one for rotation. This allows us to rotate the cathode as it engages the spring, thereby reducing the longitudinal force require to insert the cathode, moreover it also allows overcoming the static friction of the translation handle by rotating it without affecting the angular position of the cathode and the pincer.

Manipulator E moves a thickness monitor (microbalance) in and out of the coating path for calibration of the deposition rates of the Cs and Te sources.

\section{APPARATUS OPERATION}

\section{Coating operation}

The coating of the Molybdenum Cathodes with $\mathrm{Cs}_{2} \mathrm{Te}$ is studied in the papers by di Bona et $\mathrm{al}^{3}$ and by Michelato et $\mathrm{al}^{10}$, where a resulting recipe is described. Inside the MDC chamber there is a stainless steel structure with ceramic insulators in which there are installed side by side two evaporation sources, one for tellurium and the other for cesium. The sources can be heated independently via electric current. The structure has a mask in front of the cathode flat surface (when in coating position) and allows for the establishment of an electric field to collect photo-electrons. It also allows for the illumination of the cathode flat surface with ultraviolet light from an external source through a quartz window on one of the chamber ports. Figure 5 presents a schematic of the system indicating on the right side the optical bench arrangement, in the center the internal components, on the left feedthroughs disposition and around the chamber the typical connections to electronic hardware used for coating and measurement of quantum efficiency.

The evaporation sources are commercial ${ }^{11} \mathrm{Ni}$ boats, one filled with $99.999 \%$ pure Te powder and the other with a mixture of $\mathrm{Cs}_{2} \mathrm{CrO}_{4}$ and $84 \% \mathrm{Zr}-16 \% \mathrm{Al}$ (ST 101) nonevaporable getter alloy, the latter is a commercial item from the boat manufacturer. Since the position of these sources cannot be properly indicated in a schematic like Figure 5 , we include Figure 6 with sources cross-section details as an insert.

The heating currents are adjusted to yield the evaporation rate that deposits $1.0 \mathrm{~nm} / \mathrm{min}$ on the thickness monitor ${ }^{12}$ positioned in front of the cathode place by means of the $\mathrm{E}$ manipulator.

With manipulator $\mathrm{C}$, the halogen heating bulb and the thermocouple are inserted into their positions in the cathode. At this stage, the cathode is held by the pincer connected to manipulator D about 1" away from the mask. A feedback system reading the thermocouple and powering the halogen bulb controls the temperature of the cathode. It is used to slowly raise or lower the temperature of the cathode, at a rate that should be kept to less than $4.5^{\circ} \mathrm{C} / \mathrm{min}$ (usually $2.7^{\circ} \mathrm{C} / \mathrm{min}$ ) and maintain the cathode at $120^{\circ} \mathrm{C}$ during the coating. With this system the cathode is first heated close to $500^{\circ} \mathrm{C}$ for cleaning (degassing) and then held at $120^{\circ} \mathrm{C}$ during the coating. More specifically, the programmed heating schedule consists of a 3 hours linear rise followed by an 1 hour plateau at $500^{\circ} \mathrm{C}$ followed by a 3 hour linear drop to $120^{\circ} \mathrm{C}$.

Once the thickness monitor is retracted, the cathode at $120^{\circ} \mathrm{C}$ with the heating bulb and thermocouple is placed in the coating position by means of manipulator D. An ultraviolet light source is used to illuminate the cathode through the quartz window, and an anode at $+200 \mathrm{~V}$ collects photo-electrons. The photo-current is monitored with a pico-ammeter during the coating operation. First, a 10 minute exposure to the heated Te source deposits a $10 \mathrm{~nm}$ layer of Te. Then, while the cathode is illuminated by $253.7 \mathrm{~nm}$ light, the Cs is deposited at the $1.0 \mathrm{~nm} / \mathrm{min}$ rate, and the resulting photo-current is recorded in a Labview 
chart recorder. This recording shows an ascending series of steps, reaching a maximum at about 70 minutes into the Cs deposition, at which time the deposition is stopped in order to achieve the maximum quantum efficiency, and the cathode is cooled to room temperature.

\section{Quantum efficiency measurement}

The measurement of the quantum efficiency (number of photoelectrons generated per incident photon) of the cathode requires that all photoelectrons be collected and that the illumination be calibrated and well characterized. The ultraviolet (UV) light source in use is a $100 \mathrm{~W}$ high pressure $\mathrm{Hg}$ arc lamp ${ }^{13}$ that requires a 10 min warm up. It is equipped with a condensing lens, an iris diaphragm, and a $253.7 \mathrm{~nm}$ interference filter. An optical bench is used to facilitate the calibration of the UV light power and illumination of the photocathode. Besides the UV light source, the optical bench has two kinematic mounts for a UV mirror that can be placed so as to send the light beam to the cathode or to a calibrated photo-diode at equal path length. In order to properly collect the photoelectron current, the picoammeter ${ }^{14}$ is floated to the $+200 \mathrm{~V}$ bias voltage along with wire anode, the $\mathrm{Cs}$ and Te sources. A current limiting $100 \mathrm{k} \Omega$ resistor is added to the circuit for safety. Another picoammeter at ground potential is used to read the calibrated photodiode ${ }^{15}$ current. So far, we have assumed a photo-diode calibration factor of $0.05 \mathrm{~A} / \mathrm{W}$. Care is taken to minimize the ambient light during the measurements with the photo-diode, and account for background (dark current) readings.

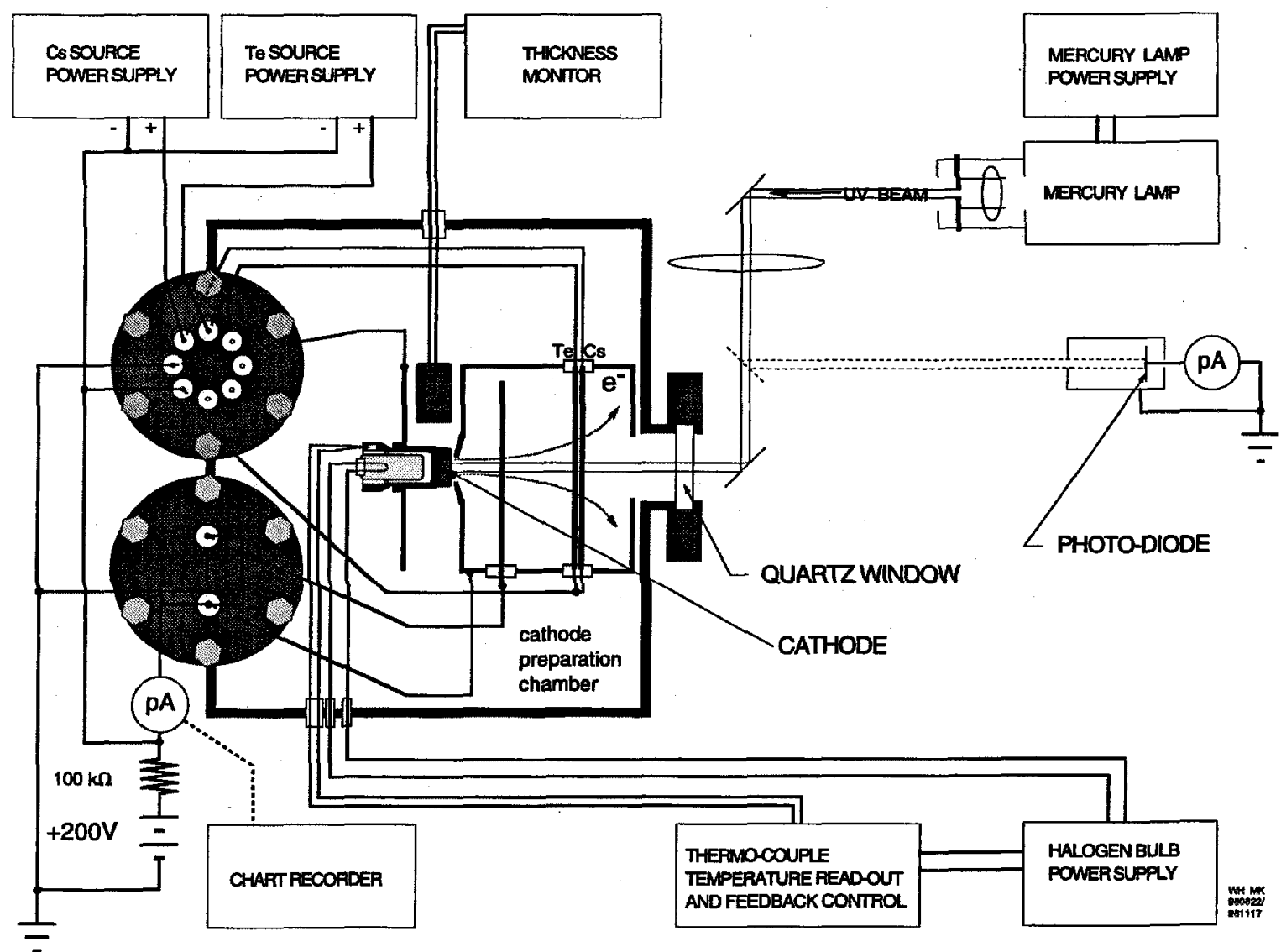

Figure 5. Photocathode preparation and QE measurement schematic. 


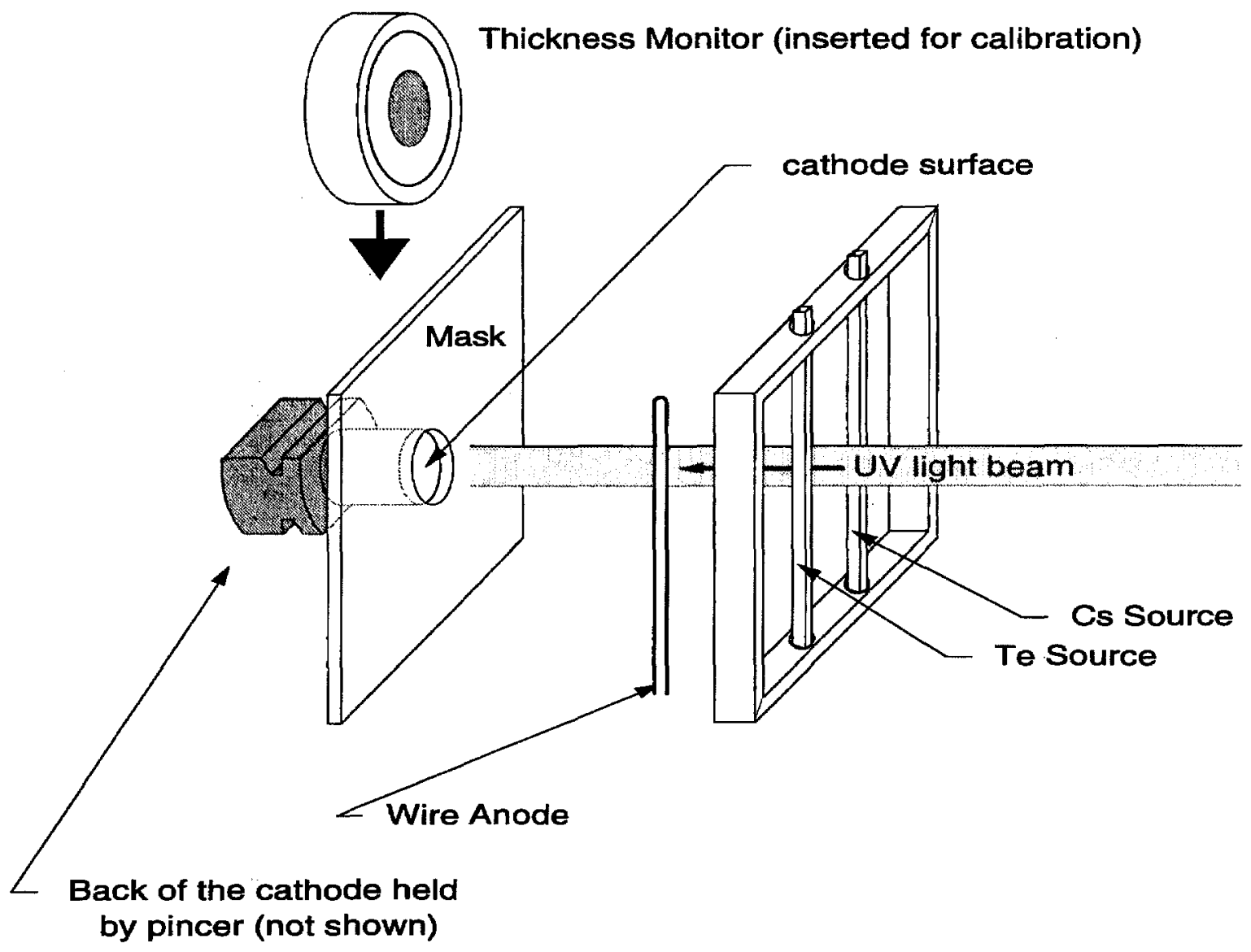

Cross-section of Sources:

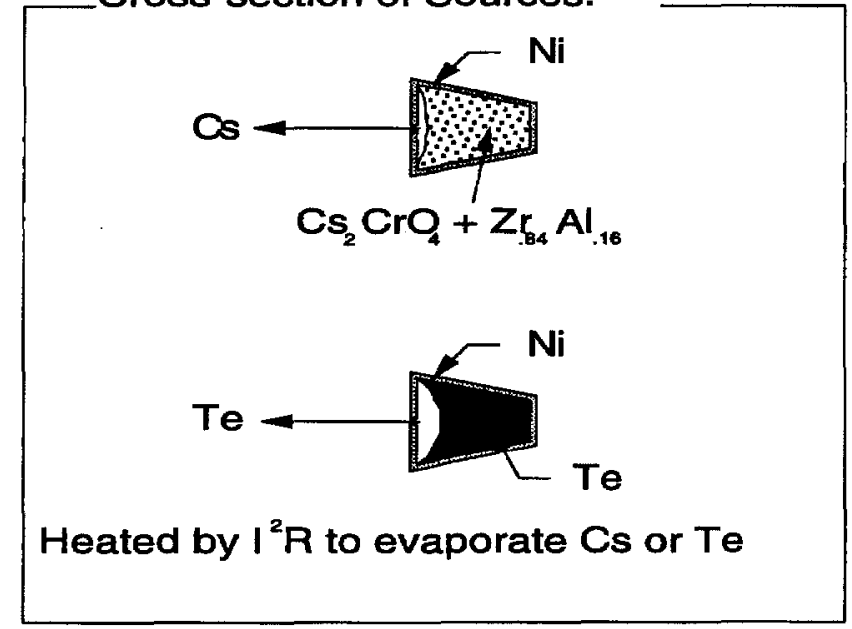

Figure 6. Photo-cathode coating geometry.

An effort to do the measurements using a light chopper and phase lock-in amplifier was hindered by the low impedance required by the photo-diode for proper reading of its 
output. The advantage of this method would be the automatic correction for the dark current and the use of the same instrument for the measurement of both currents.

'E. Chevallay, J. Durand, S. Hutchins, G.Suberlucq, M.Wurgel, "Photocathodes tested in the DC gun of the CERN photoemission laboratory", Nuclear Instruments \& Methods in Physics Research A340(1994) $146-156$

${ }^{2}$ S.H. Kong, D.C. Nguyen, R.L. Sheffield and B.A Sherwood, "Fabrication and characterization of Cesium Telluride photocathodes: A promising electron source for the Los Alamos Advanced FEL", Nuclear Instruments \& Methods in Physics Research A358(1995) 276-279.

${ }^{3}$ A. di Bona, F. Sabary, S. Valeri, P. Michelato, D. Sertore and G. Suberlucq, “Auger and X-ray photoemission spectroscopy on Cs2Te photocathodes", J. Appl. Phys. 80, 3024 (1996)

${ }^{4}$ P. Michelato, "Photocathodes for RF photo injectors", to be published in the Proceedings of the 1996 Free Electron Laser Conference in Rome.

${ }^{5}$ P. Michelato, D. Sertore, C. Pagani, A. di Bona, S. Valeri, G. Ferrini and F. Parmigiani, “Cs2Te photocathode for the TTF Injector II', Proceedings of the European Particle Accelerator ConferenceBarcelona 1996

${ }^{6}$ Surface Science Analysis Chamber- Model SSAC-12D - Manufactured by MDC -23842 Cabot Boulevard, Hayward, CA, 94545-1651 - Tel.(510)887-6100

URL: www.mdc-vacuum.com

${ }^{7}$ Magnetic Drive Model ZMLRV209 - Manufactured by Vacuum Generators - Maunsell Road, Hastings, E. Sussex, England, TN389NN - Tel. 441424853553

URL: www.vacgen.com

${ }^{8}$ Linear-Rotary Transfer Feedthrough Model VFS-1690-36-R - Manufactured by Huntington Laboratories Inc., 1040 L'Avenida, Mountain View, CA 94043, USA, Tel. (415)964-3323

URL: www.huntvac.com

${ }^{9} \mathrm{C}$, D and $\mathrm{E}$ are Linear Transfer Mechanisms (LTM Series-manual versions) - Manufactured by Vacuum Generators - Maunsell Road, Hastings, E. Sussex, England, TN389NN - Tel. 441424853553

URL: www.avem.org/VG.html

${ }^{10}$ P. Michelato, C. Pagani, D. Sertore, A di Bona, S. Valeri "Characterization of Cs2Te photoemissive film: formation, spectral responses and pollution. presented at the 1996 FEL Conference in Rome.

${ }^{11}$ Saes Getters - Viale Italia 77, 20020 Gainate (MI) Italy - Fax +39-2-93178320 Saes Getters/USA Inc. 1122 E. Cheyenne Mountain Blvd., Colorado Springs, CO 80906 USA Tel (303)576-3200

URL: www.saesgetters.com

${ }^{12}$ Thickness Monitor Model STM-100/MF - Manufactured by Sycon Instruments Inc - 6757 Kinne Street, East Syracuse, NY 13057-1215, USA, Tel. (315)463-5297

URL: www.sycon.com

${ }^{13}$ Mercury Lamp Model 6281 -.Manufactured by Oriel Corp. of America -250 Longbeach Blvd., Stratford, CT 06497, USA, Tel. (203)377-8282

URL: http://www.oriel.com

${ }^{14}$ Picoammeters: Model 486 used for the cathode and Model 417 used for the photodiode, both manufactured by Keithley Instruments, Inc. -28775 Aurora Road, Cleveland, OH 44139, USA, Tel. (440)248-0400

URL: www.keithley.com

${ }^{15}$ Model RS 564-043 ECI 